\title{
A Novel Passivity Based Control of Active Magnetic Bearing Systems without Conventional Cross-Feedback*
}

\author{
Satoru SAKAI ${ }^{* *}$, Kenta KURIYAMA ${ }^{* *}$ and Kenzo NONAMI** \\ ${ }^{* *}$ Chiba Univeristy, Department of Engineering \\ 1-33, Inage-ku, Chiba 263-8522, Japan \\ E-mail: satorusakai@gakushikai.jp
}

\begin{abstract}
This paper gives a new passivity based control of active magnetic bearing systems. First we give a new modeling in port-Hamiltonian form of flywheel systems. Second, we give a new passivity based control which does not have any canceling term for the gyroscopic effect unlike the conventional cross-feedback control, and also does not have any gains to make the closed-loop unstable unlike PID feedback control. In this paper, the linear control is especially studied from the viewpoint of comparisons. Finally we give some simulation and experimental results and confirm the validity of the control methodology in the presence of unbalance influence.
\end{abstract}

Key words : Rotor Dynamics, Passivity, Casimir Functions

\section{Introduction}

This paper discusses a new passivity based control of active magnetic bearings ${ }^{(16)}$ such as flywheel systems in Fig. 1. The flywheel systems are contact-free rotating bodies levitated and controlled by magnetic forces and one of the energy storing elements whose rotational kinetic energy is almost conserved or is transformed to the electrical energy to drive milling spindles, satellites, power plants or electric automobiles and so on ${ }^{(19)(20)(21)(22)}$.

The many control problems of flywheel systems are from the (inertia) unbalance ${ }^{(18)(17)}$. The non-uniform mass distribution loses the stability or the control performance such as the response of the (contact-free) rotational-axis orientation.

In order to reduce the unbalance influence, cross-feedback, that is, feedback canceling is conventionally applied for the gyroscopic effect which depends on the rotational speed and is clearly one of the structural properties of flywheel systems. The cross-feedback makes it possible to introduce many fruitful results from linear systems and control theory ${ }^{(13)(14)}$ while this feedback canceling does not work well without identifying exact parameters, such as the center of gravity and the moment of inertia matrix, precisely. The linear robust controllers against the modeling errors requires high computational costs in general.

On the other hand, passivity based control ${ }^{(2)(10)(11)}$, which is one of the recent trends of the nonlinear control, utilizes the physical and structural properties for modeling ${ }^{(5)}$ and control $^{(4)}$ without or with less feedback canceling. This brings some advantages from the viewpoints of robustness, computational costs and re-design and so on. For example, in case of robotic manipulators, it is well-known that the global asymptotic stability is achieved without canceling the nonlinearity such as the centrifugal effect and Coriolis effect ${ }^{(2)}$ by the passivity based control.

In this paper, first, we give a new modeling in port-Hamiltonian form of flywheel systems. Second, we give a new passivity based control which achieves the global asymptotic stability while NOT canceling the gyroscopic effect at all. Finally we give some simulation 


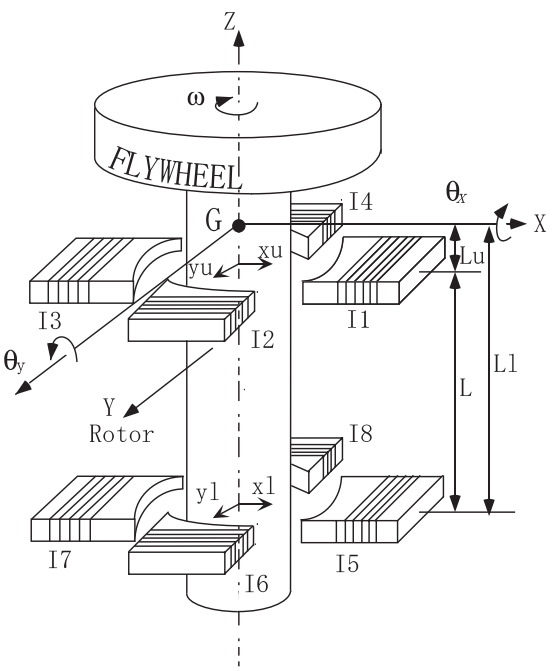

Fig. 1 Flywheel system.

and experimental results and conclude this paper.

\section{Preliminary}

In this section, we refer basic concepts and tools such as passivity, port-Hamiltonian systems and their control methods.

\subsection{Passivity}

The concept of passivity is not quite new and the passivity based control is from the work of Takegaki and Arimoto ${ }^{(2)}$. However, this methodology is recently developed in a new framework and more fruitful results are obtained.

Let us consider a state space system

$$
\Sigma\left\{\begin{aligned}
\dot{x} & =f(x, u), u \in U \\
y & =h(x, u), y \in Y
\end{aligned}\right.
$$

where $x$ is a local coordinates for a finite dimensional manifold, $U$ and its dual space $U^{*}=Y$ are a finite-dimensional linear spaces.

Definition $\mathbf{1}^{(10)}$ The system $\Sigma$ is said to be passive with respect to the supply rate $s(u, y)=$ $u^{\mathrm{T}} y$ if there exists a $C^{1}$ (continuously differentiable) storage function $S(x) \geq 0$ such that

$$
\frac{d S}{d t} \leq s(u, y)
$$

for all $x$ and $u$.

In this paper, some dimension of the vectors and matrices are omitted because of the uniqueness.

\subsection{A Special Class of Port-Hamiltonian systems}

Definition $2^{(10)}$ A (simplified version of) port-Hamiltonian system with a Hamiltonian $H(x) \in \mathbf{R}$ is a system described by

$$
\left\{\begin{array}{l}
\dot{x}=J(x) \frac{\partial H(x)}{\partial x}^{\mathrm{T}}+g(x) u \\
y=g(x)^{\mathrm{T}}{\frac{\partial H(x)^{\mathrm{T}}}{\partial x}}^{\mathrm{T}}
\end{array}\right.
$$


with $u, y \in \mathbf{R}^{m}, x \in \mathbf{R}^{n}$ and a skew symmetric matrix $J(x)$, i.e. $-J(x)=J(x)^{\mathrm{T}}$ holds. The following property of such systems is known.

Lemma $1^{(10)}$ Consider the port-Hamiltonian system (2). Suppose the Hamiltonian $H(x)$ satisfies $H(x) \geq H(0)=0$. Then the input-output mapping $u \mapsto y$ of the system is passive with respect to the storage function $H$, that is, $\dot{H} \leq u^{\mathrm{T}} y$ and the feedback

$$
u=-D y
$$

with a matrix $D=D^{\mathrm{T}} \geq 0 \in \mathbf{R}^{m \times m}$ renders $(u, y) \rightarrow 0$. Furthermore if $H(x)$ is positive definite and if the system is zero-state detectable, then the feedback (3) renders the origin asymptotically stable.

The zero-state detectability and the positive definiteness of the Hamiltonian assumed in Lemma 1 do not always hold for general port-Hamiltonian systems. In this case, the generalized canonical transformation ${ }^{(9)}$ provides a stabilization method which is a generalization of the virtual potential energy ${ }^{(2)}$.

Furthermore, the other control methodologies can be applied if the port-Hamiltonian systems have the following property ${ }^{(10)(7)}$.

Lemma 2 Suppose that the (integrable) port-Hamiltonian system (2) has Casimir functions with respect to $J$. Then

$$
\frac{\partial C(x)}{\partial x} J=0
$$

holds for any Hamiltonian at the input $u=0$.

\section{MODELING}

In this section, we give a new modeling in port-Hamiltonian form ${ }^{(1)}$ of flywheel systems. The state-space equations of flywheel systems is usually given based on the rotor dynamics

$$
\begin{aligned}
& m \ddot{x}_{g}=u_{1} \\
& m \ddot{y}_{g}=u_{2} \\
& I_{d} \ddot{\theta}_{x}=+\omega_{3} I_{p} \dot{\theta}_{y}+u_{3} \\
& I_{d} \ddot{\theta}_{y}=-\omega_{3} I_{p} \dot{\theta}_{x}+u_{4}
\end{aligned}
$$

where $m$ is the rotor mass, $I_{d}, I_{p}$ are the rotor inertia, $x_{g}, y_{g}$ are the rotor (the center of gravity) displacements, $\theta_{x}, \theta_{y}$ are the rotor angles, $u_{i}(i=1, \ldots, 4)$ are the control inputs. Figure 1 shows the (normal) coordinates of the flywheel systems ${ }^{(15)}$.

In this paper the stability is discussed on the origin $\left(x_{g} y_{g} \theta_{x} \theta_{y}\right)=0$ which is the equilibria of the rotor dynamics.

Remark The rotor dynamics is not the rigid body dynamics. The third and the forth equations (7), (8) are an approximation in the following sense at least. First, the equations are not the rigid body dynamics which should be discussed not on $\mathbf{R}^{3}$ but on $S O$ (3) or should be discussed as the cascaded form ${ }^{(1)}$. Second, the gains of input $u_{3}$ and $u_{4}$ should depend on the flywheel orientation since these torques are generated from AMB forces fixed in the inertia coordinate frame. This means that $\dot{\omega}_{3}$ actually depends on input $u_{3}$ and $u_{4}$. In all, the rotor dynamics is an approximation of the rigid body dynamics and many properties (such as modeling) of the rigid body dynamics may not hold for the rotor dynamics. 
Theorem 1 (Modeling) Consider the rotor dynamics (5)-(6) and the coordinate transformation

$$
\left\{\begin{array}{l}
q=(s, \theta)^{\mathrm{T}} \\
p=M(\dot{s}, \dot{\theta})^{\mathrm{T}}
\end{array}\right.
$$

where $s=\left(x_{g}, y_{g}\right) \in \mathbf{R}^{2} \theta=\left(\theta_{x}, \theta_{y}\right) \in \mathbf{R}^{2}$ and $M=\operatorname{diag}\left(m, m, I_{d}, I_{d}\right) \in \mathbf{R}^{4 \times 4}$. Then, if the output is taken as the velocity $\dot{q}$, the rotor dynamics (5)-(8) are modeled in the portHamiltonian form

$$
\Sigma_{a m b}\left\{\begin{array}{l}
\dot{x}=\underbrace{\left[\begin{array}{cc}
0 & I_{4} \\
-I_{4} & S_{4}
\end{array}\right]}_{J_{a m b}}{\frac{\partial H^{\mathrm{T}}}{\partial x}}^{\mathrm{T}}+\left[\begin{array}{c}
0 \\
I
\end{array}\right] u \\
y=\frac{\partial H}{\partial p}^{\mathrm{T}}
\end{array}\right.
$$

where $x=(q, p) \in \mathbf{R}^{8}, H=(1 / 2) p^{\mathrm{T}} M^{-1} p$ and

$$
S_{4}\left(\omega_{3}\right)=\left[\begin{array}{cc}
0 & 0 \\
0 & S_{2}\left(\omega_{3}\right)
\end{array}\right], \quad S_{2}\left(\omega_{3}\right)=\left[\begin{array}{cc}
0 & +\omega_{3} I_{p} \\
-\omega_{3} I_{p} & 0
\end{array}\right]
$$

Proof of Theorem 1 Eq.(10) can be derived by a direct calculation by the transformation. Since $S_{2}^{\mathrm{T}}+S_{2}=0$ holds for any $\omega_{3}, S_{4}$ and $J_{a m b}$ are skew-symmetric matrices, that is, $-J_{a m b}\left(\omega_{3}\right)=J_{a m b}\left(\omega_{3}\right)^{\mathrm{T}}$. These imply that the system (10) is a port-Hamiltonian system with the Hamiltonian $H$ bounded from below. (Q.E.D.)

\section{Control}

In this section, we give some passivity based controls without conventional cross-feedback based on the previous sections. First, as the simplest case, we give a static control to achieve the global stability of flywheel systems. Second, we give a dynamic one in the presence of disturbances.

\subsection{Static control without cross-feedback against the gyroscopic effect}

Theorem 2 (Static global stabilization) Consider the system $\Sigma_{a m b}$ and the following linear controller

$$
u=-K_{q} q-D \dot{q}
$$

with any positive definite matrix $K_{q}=K_{q}^{\mathrm{T}}>0$ and $D=D^{\mathrm{T}}>0$. Then the equilibrium set of the closed-loop system contains only the origin and it is globally asymptotically stable.

Remark The industrial (diagonal) PD controller is a special case of the above controller. This makes the proposed controller different from the other similarly structured or other passivity based controllers ${ }^{(23)}{ }^{(24)}$. Note that there is NO cross-feedback term as

$$
u=-K_{q} q-D \dot{q}+\underbrace{\left[\begin{array}{cc}
0 & 0 \\
0 & S_{2}\left(\omega_{3}\right)
\end{array}\right] \dot{q}}_{\text {cross-feedback }}
$$

in Eq.(12), that is, the gyroscopic effect is not canceled any more. 


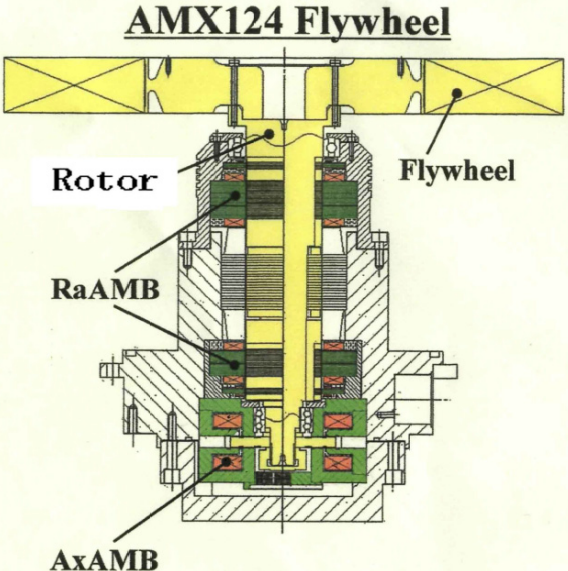

Fig. 2 Flywheel.

Proof of Theorem 2 By applying the first term of the controller (12), the original system $\Sigma_{a m b}$ is converted to the following system with new input and output

$$
\left\{\begin{aligned}
{\left[\begin{array}{c}
\dot{q} \\
\dot{p}
\end{array}\right] } & =\left[\begin{array}{cc}
0 & I_{4} \\
-I_{4} & S_{4}
\end{array}\right]\left[\begin{array}{c}
\frac{\partial(H+U)}{\partial q} \\
\frac{\partial(H+U)}{\partial p}
\end{array}\right]+\left[\begin{array}{l}
0 \\
I
\end{array}\right] \bar{u} \\
\bar{y} & =\frac{\partial(H+U)}{\partial p}^{\mathrm{T}}
\end{aligned}\right.
$$

where $U=(1 / 2) q^{\mathrm{T}} K_{q} q$ is the virtual energy. Since this is also a port-Hamiltonian system ${ }^{(9)(4)}$, from Lemma 1, the state converses to the set

$$
\Omega_{s} \equiv\{\bar{y}=\bar{u}=0\}
$$

by applying the second term of the controller (12). Here, the input-output map of the system (13) is zero-state detectable, that is, in the set $\Omega_{s} \bar{y} \equiv 0 \Leftrightarrow \dot{q} \equiv 0 \Rightarrow \ddot{q} \equiv 0 \Rightarrow q \equiv p \equiv 0$. (Q.E.D.)

Note that the time-varing gains such as $K_{q}\left(\omega_{3}(t)\right)$ and $D\left(\omega_{3}(t)\right)$ are treated in Theorem 2 and that the stabilizer (12) is robust for any perturbed inertia matrix $M+\delta M>0$.

\subsection{Dynamic control without cross-feedback against the gyroscopic effect}

In this subsection, we discuss the global stability of a dynamic passivity based control without conventional cross-feedback.

Theorem 3 (Dynamic global stabilization) Consider the system $\Sigma_{a m b}$ and the following linear controller

$$
\left\{\begin{aligned}
\dot{r} & =X K_{q} q \\
u & =-K_{q} q+S_{4} X^{\mathrm{T}} L(X p+r)-D\left(\dot{q}+X^{\mathrm{T}} L(X p+r)\right) .
\end{aligned}\right.
$$

with any positive definite matrix $L=L^{\mathrm{T}}=\operatorname{diag}\left(L_{s}, L_{\theta}\right)$ and any positive definite matrix $X=\operatorname{diag}\left(X_{s}, X_{\theta}\right)$. Then the equilibrium set of the closed-loop system contains only the origin and it is globally asymptotically stable.

Proof of Theorem 3 By applying the controller output (15) without the third term, the closed-loop system is the following system with new input and output 


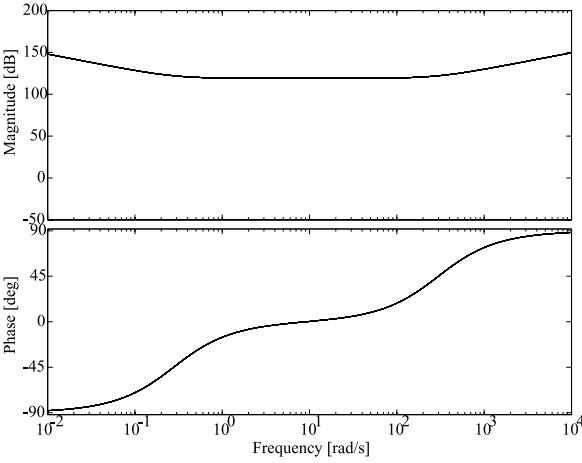

Fig. 3 Bode-plots of $(1,1)$ element

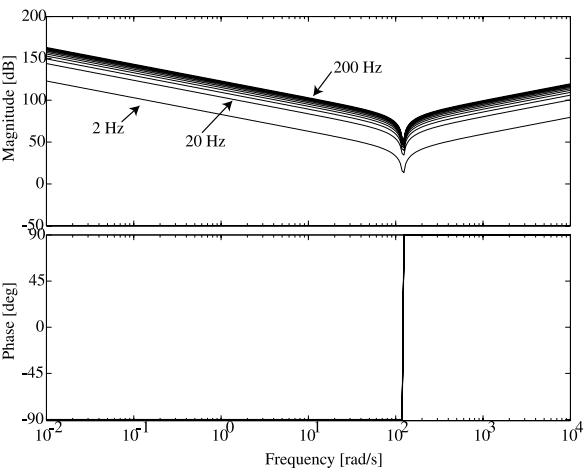

Fig. 5 Bode-plots of $(1,3)$ element

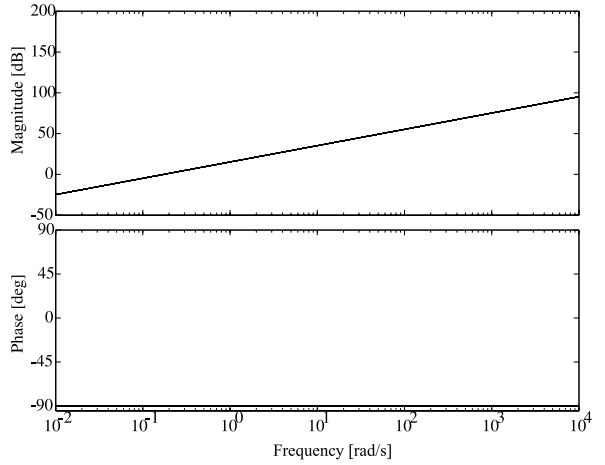

Fig. 4 Bode-plots of $(1,2)$ element

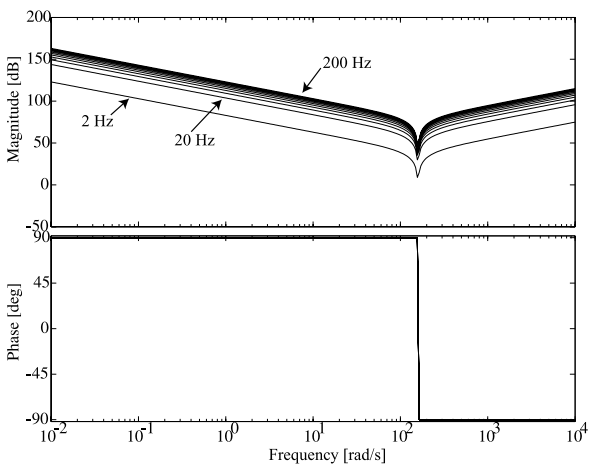

Fig. 6 Bode-plots of $(1,4)$ element

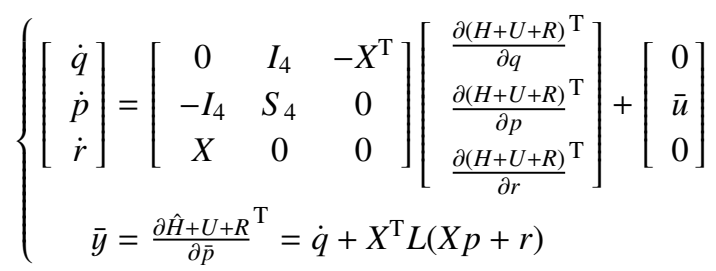

where

$$
R=\frac{1}{2}\left(X_{\theta} p_{\theta}+r_{\theta}\right)^{\mathrm{T}} L_{\theta}\left(X_{\theta} p_{\theta}+r_{\theta}\right)
$$

is a lower bounded function and the Casimir function

$$
C_{s}(z), z=X_{s} p_{s}+r_{s}
$$

exists from the PDE (4) where $p=\left(p_{s}, p_{\theta}\right)^{\mathrm{T}}, r=\left(r_{s}, r_{\theta}\right)^{\mathrm{T}}$.

By considering the time-derivative of a (Lyapnov candidate) function

$$
H+U+R+\frac{1}{2}\left(z-z_{d}\right)^{\mathrm{T}} L_{s}\left(z-z_{d}\right)
$$

with the desired value $z_{d}=z(0)$, based on Lemma 1 and the previous result ${ }^{(7)}$, the state converses to the set $\Omega_{d} \equiv\{\bar{y}=\bar{u}=0\}$ by the third term of the controller output (15). In the set $\Omega_{d}$

$$
\begin{aligned}
& M^{-1} p+X^{\mathrm{T}} L(X p+r) \equiv 0 \\
& \Rightarrow M^{-1} \dot{p}+X^{\mathrm{T}} L(X \dot{p}+\dot{r}) \equiv 0 \\
& \Rightarrow\left(M^{-1}+X^{\mathrm{T}} L X\right)\left(-K_{q} q\right)+M^{-1} S_{4} \cdot 0+X^{\mathrm{T}} L\left(-X K_{q} q\right)=0 \Rightarrow q \equiv p \equiv 0 .
\end{aligned}
$$




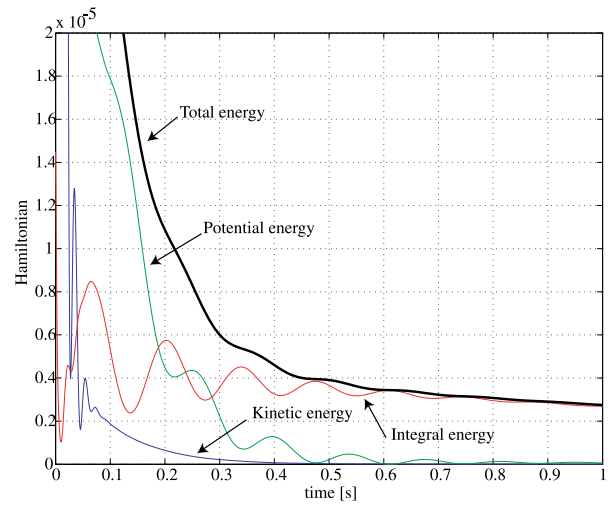

Fig. 7 Hamiltonian $(110 \mathrm{~Hz}$, no unbalance $)$

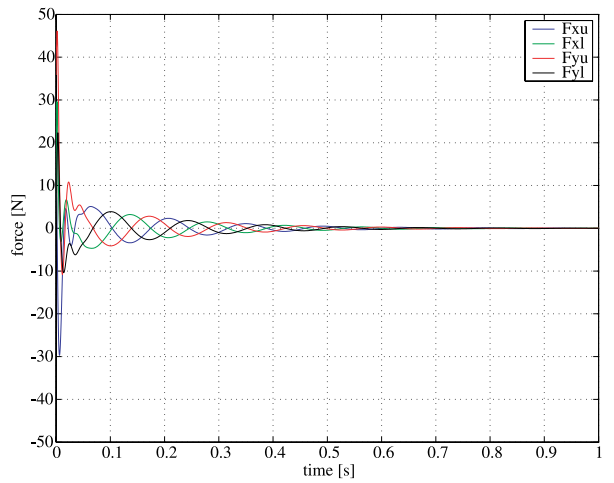

Fig. 9 Input forces $(110 \mathrm{~Hz}$, no unbalance)

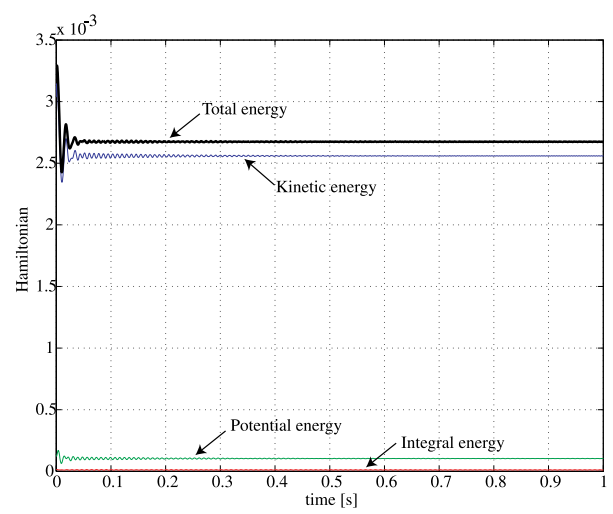

Fig. 8 Hamiltonian $(110 \mathrm{~Hz}$, with unbalance $)$

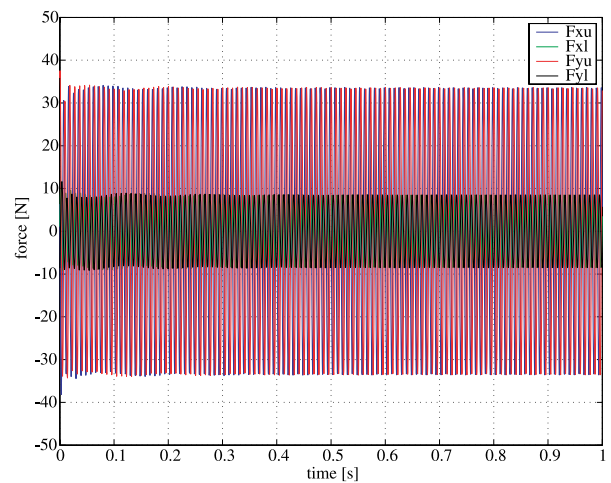

Fig. 10 Input forces $(110 \mathrm{~Hz}$, with unbalance)

Furthermore this implies $X^{\mathrm{T}} L r \equiv 0$, that is, $r \equiv 0$. (Q.E.D.)

Remark Every term in Eq.(15) can be time-varying and the proposed linear feedback has a very simple structure with many free design parameters. In a special case of $X K_{q}=I$, the above controller output is rewritten as

$$
u=-K_{q} q-K_{L} r-D \dot{q}+S_{4}\left(\bar{K}_{L} r+\bar{D} \dot{q}\right)
$$

where $\bar{K}_{L}$ and $\bar{D}$ are determined from the other parameters and the disturbance rejection is easily confirmed. Without the fourth term of Eq.(20), the closed-loop system can be easily unstable by badly tuned gains in the presence of unbalance or perturbation. The controller (20) with the fourth term achieves the global asymptotic stability for ANY (high) gain $K_{q}$, $K_{L}$ and $D$. The fourth term differs from the cross-feedback term which depends on physical parameters only. These lead to not only the low computational costs but also the robustness of the closed-loop system as shown in the next section.

\section{Simulation and Experiment}

Figure 2 shows the cross-section of flywheel systems in the experiment. The mass is $13.67 \mathrm{~kg}$ and the diameter is $400 \mathrm{~mm}$. The parameters are $I_{p}=0.0179, I_{d}=0.0180$. The gains are $K_{q}\left(=X^{-1}\right)=3 \times 10^{3} I_{4}, D=0.9 \times 10^{6} I_{4}, L=0.25 \times 10^{6} I_{4}$ and generate a linear controller whose bode plots are shown in Figs. 3-6. In this case, the transfer function matrix of the controller is symmetric and the $(1,2),(1,3),(1,4)$ elements are equal to the $(3,4),(2,3),(2,4)$ elements, respectively. Unlike with the linear robust controllers (e.g. $H_{\infty}$ or $\mu$-controllers), the gains are still high in the high-frequency region and modulated by the rotational speed $\omega_{3}$ (from $2 \mathrm{~Hz}$ to $200 \mathrm{~Hz}$ ). 


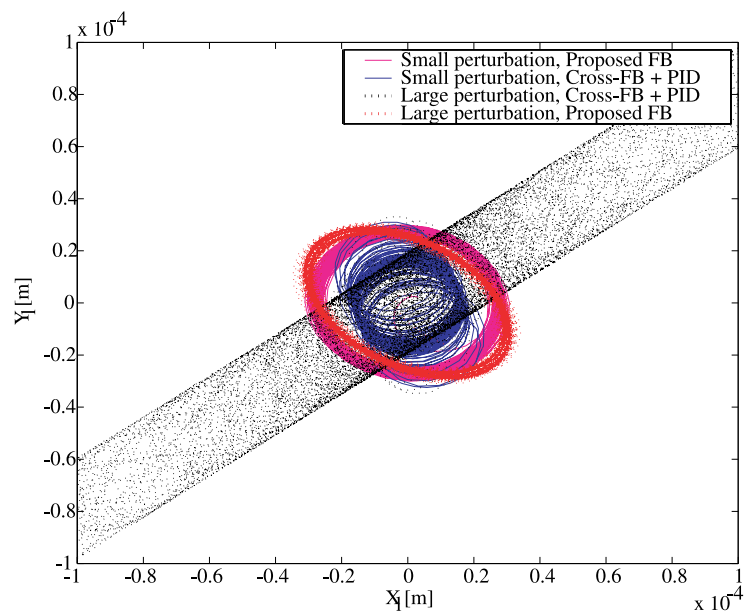

Fig. 11 Robust stability $(110 \mathrm{~Hz}$, with unbalance and perturbation, simulation)

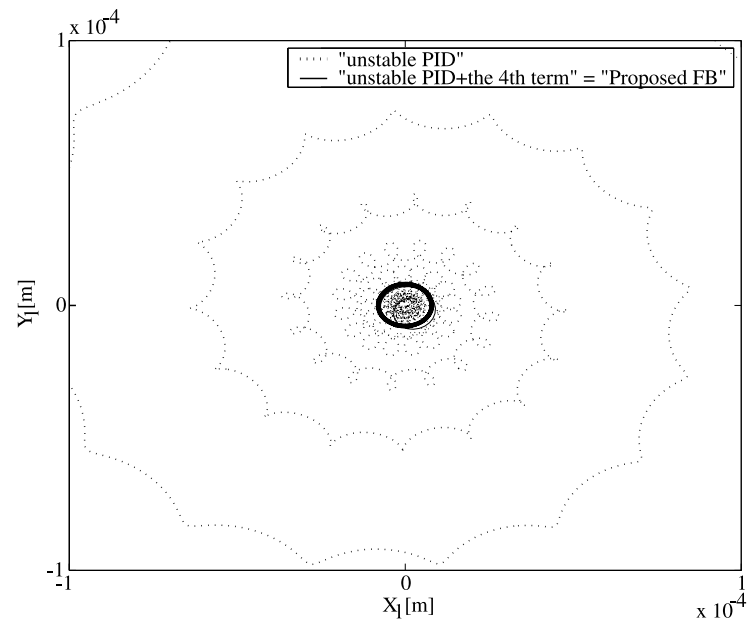

Fig. 12 Effectiveness of the fourth term in Eq.(20) $(110 \mathrm{~Hz}$, by unstable gain)

Figure 7 shows the time response of the closed-loop Hamiltonian in simulation for $\omega_{3}$ $=110 \mathrm{~Hz}$. It is confirmed that the closed-loop Hamiltonian is monotonically decreasing and tends to 0 . Not only the kinetic energy $H(t)$ but also the potential energy $U(t)$ and the additional energy $R(t)+(1 / 2) z^{T} L_{s} z$ converse to 0 . Figure 8 shows the time response of the closed-loop Hamiltonian in the presence of the unbalance influence which is modeled ${ }^{(25)}$ as

$$
\begin{aligned}
m \ddot{x} & =u_{1}+M r_{c} \omega_{3}^{2} \cos \left(\omega_{3} t\right) \\
m \ddot{y} & =u_{2}+M r_{c} \omega_{3}^{2} \sin \left(\omega_{3} t\right) \\
I_{d} \ddot{\theta}_{x} & =+\omega_{3} I_{p} \dot{\theta}_{y}+u_{3}+l_{c} M r_{c} \omega_{3}^{2} \cos \left(\omega_{3} t\right) \\
I_{d} \ddot{\theta}_{y} & =-\omega_{3} I_{p} \dot{\theta}_{x}+u_{4}+l_{c} M r_{c} \omega_{3}^{2} \sin \left(\omega_{3} t\right) .
\end{aligned}
$$

with $l_{c}=1 \times 10^{-4}$ and $r_{c}=2 \times 10^{-5}$. The total Hamiltonian converses to a non-zero value, which implies the stability of the closed-loop system. Figure 9 and Fig. 10 show the input forces, respectively. There are no input saturations in both cases.

Figure 11 shows the comparison of robust stability for inertia perturbations between the proposed feedback and and the conventional cross-feedback with PID feedback. There are two cases, that is, a case of small perturbation (1\% perturbation of the inertia moment matrix) and a case of large perturbation (10\% perturbation of the inertia moment matrix). In both cases, 

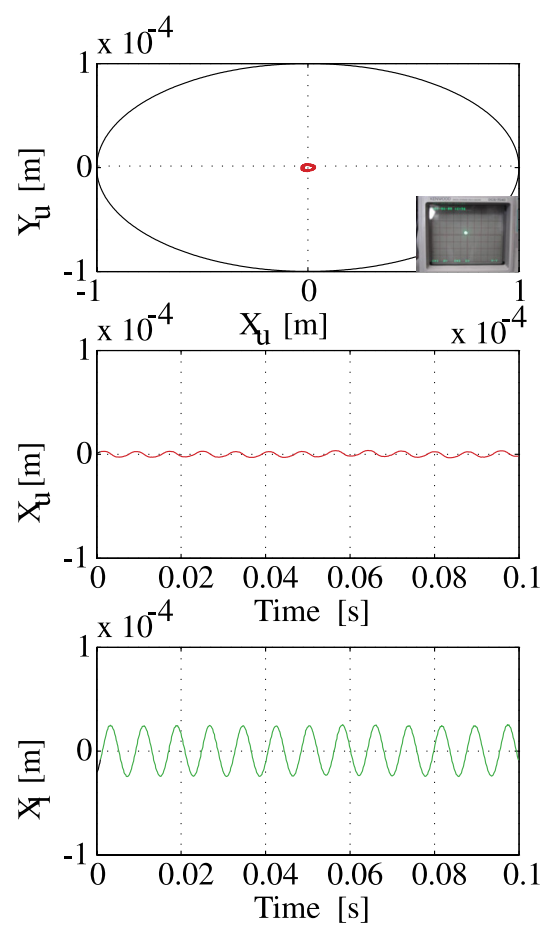
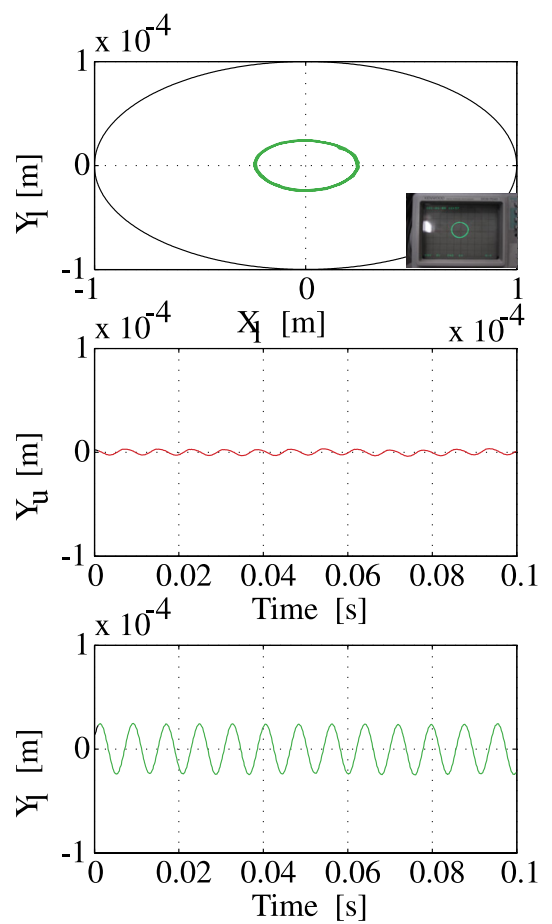

Fig. 13 Robust stability (110Hz, by bad gain tuned for $2 \mathrm{~Hz}$, experiment)

the perturbed inertia moment matrix has non-zero values in non-diagonal parts as well and corresponds to the unbalance influence. Even though the PID gains for the cross-feedback are well-tuned for the small perturbation, the response of the cross-feedback with the PID gains become unstable in case of the large perturbation, while that of the proposed feedback is still stable and has the almost same behavior.

Figure 12 shows the effectiveness of the fourth term in Eq.(20). Even if the gains $K_{q}$, $K_{L}$ and $D$ are badly tuned so that the response of the PID feedback is unstable, the response of the proposed feedback, that is, the same PID feedback with the fourth term (determined from the other parameters) is stable. Figures 3-6, Fig. 11 and Fig. 12 imply that the proposed feedback is essentially different from many conventional feedbacks, such as the family of $H_{\infty}$ feedback, the cross-feedback and the (diagonal) PID feedback. In order to demonstrate these differences, the linear controller is proposed and discussed in this paper (though many nonlinear extensions will achieve the stability and control performance).

Figure 13 shows the experimental results in $X_{u}-Y_{u}$ plane and $X_{l}-Y_{l}$ plane for $110 \mathrm{~Hz}$. The gains $K_{q}, K_{L}$ and $D$ are BADLY tuned, that is, are tuned for the different rotational speed in order to confirm the robust stability while keeping the experimental safety. The sampling frequency was $8000 \mathrm{~Hz}$. In this case, the gains were very baddly tuned as the PID feedback for just $2 \mathrm{~Hz}^{(25)}$ and the fourth term in Eq.(20) was not modulated. It is confirmed that the rotor position is still smoothly stabilized for $110 \mathrm{~Hz}$ (from $2 \mathrm{~Hz}$ actually). The rotor velocities are also smoothly stabilized and a natural periodic motion is generated.

In all, the validity of the proposed modeling and control methodology is confirmed by the simulation and the experiment. Many (classical) existing gain-tuning methods ${ }^{(6)}$ can be applied because the proposed controllers are just linear systems.

\section{Conclusion}

This paper gives a new modeling and passivity based control of active magnetic bearing systems. Unlike with the cross-feedback, the proposed controllers have NO canceling terms of the gyroscopic effect. Unlike with PID feedback, the proposed controllers have NO gains 
to make the closed-loop unstable. First we give a new modeling in port-Hamiltonian form of flywheel systems. Second, we give a new static and dynamic passivity based control which achieves the global asymptotic stability without the conventional cross-feedback. In this paper, the linear control is especially studied from the viewpoint of comparisons. Finally we give simulation and experimental results and confirm the validity of the proposed modeling and control methodology in the presence of unbalance influence. The simplicity of the proposed controllers brings both low computational costs and high robustness while keeping the extendability.

\section{References}

( 1 ) V. I. Arnold: Mathematical Methods of Classical Mechanics, Springer-Verlag, New York, 1989.

( 2 ) S. Arimoto: Control theory of nonlinear mechanical systems, Clarendon press / oxford, 1996.

( 3 ) H. Khalil: Nonlinear control systems, Prentice Hall, 2002.

( 4 ) R. Ortega and Eloisa Garcia-Canseco, Interconnection and Damping Assignment Passivity-Based Control: A survey, European Journal of Control, 1-27, 2004.

( 5 ) Stramigioli S., Modeling and IPC control of interactive mechanical systems, Springer, 2001.

( 6 ) S. Sakai and K. Fujimoto, Dynamic output feedback stabilization of a class of nonholonomic Hamiltonian systems, Proc. IFAC World Congress 2005, 1967-1970, 2005.

( 7 ) S. Sakai, Control of Casimir functions for port-Hamiltonian systems, Proc. SICE CCS 2007, 92-95(063-4-2), 2007.

( 8 ) S. Sakai, Stramigioli S. Port-approaches to motion generations for mechanical systems, Proc. ICRA 2007, 92-95, 2007.

( 9 ) K. Fujimoto and T. Sugie: Canonical transformation and stabilization of generalized Hamiltonian systems, Systems \& Control Letters, 42(3), 217-227, 2001.

(10) A. J. van der Schaft: $L_{2}$-Gain and Passivity Techniques in Nonlinear Control, SpringerVerlag, 2000.

(11) B.D.O. Anderson and S. Vongpanitlerd: Network analysis and synthesis: a modern systems theory approach, , Printice Hall, 1973.

(12) R. Gasch and H. Pfutzner: Rotor dynamics, Morikita (in Japanese), 1978.

(13) K. Nonami, T. Ito: $\mu$ synthesis of flexible rotor-magnetic bearing systems IEEE Transactions on Control Systems Technology, Volume 4, Issue 5, 503 - 512, 1996.

(14) K. Zhou, J. C. Doyle, K. Glover: Robust and Optimal Control, Prentice-Hall, 1996.

(15) S. Sivrioglu, K. Nonami: Sliding mode control with time-varying hyperplane for AMB systems IEEE/ASME Transactions on Mechatronics, Volume 3, Issue 1, 51-59, 1998.

(16) H. Bleuler: A survey of magnetic levitation and magnetic bearing types, JSME International Journal, Volume 35, No.3, 1992.

(17) H. Bleuler: 20 years ISMB: Then, Now, Future, International conference of ISMB, K-1, 2008.

(18) T. Mizuno: A transfer function approach to active magnetic bearing control systems, JSME Volume 65, No.637, 1999 (in Japanese).

(19) Higuchi, T.: Magnetic bearings, Proc. 2nd Int. Symposium on magnetic bearings, Univ. of Tokyo, 1990.

(20) Allaire, P.(ed): ROMAG Conference on Magnetic Bearings and Dry Gas Seals, Washington D.C. 1991.

(21) Henrikson, C. H., Lyman, J. and Studer, P. A.: Magnetetically suspended momentum wheels for space craft stabilization, AIAA paper, 74-128, 1974.

(22) Parente, R. B.: Stability of a magnetic suspension device, IEEE trans. on Aerospace and electronic systems, Vol.Aes-5, No.3, 474, 1969.

(23) P. Tsiotas, M. Arcak: Low-Bias control of AMB subject to voltage saturation: Panagio- 
tis, T, Mstate-feedback and observer designs, IEEE trans. on control systems technology, Vol.13, No.2, Panagiotis, T, M262-273, 2005.

(24) P. Buhler, R. Larsonneur, Mecos Traxler AG: Method and appratus for controlling a magnetic bearing device, USpatent, US2007296367(A1), 2007.

(25) K. Kuriyama, K. Nonami, B. Rachmanto: Attitude stabilization control of AMBFrywheel supported by two-axes gimbals, Proc. of the 11th international symposium on magnetic bearings, 526-532, 2008. 